CERN-TH/99-364

hep-th/9911218

\title{
Solitons in Brane Worlds
}

\author{
Donam Youm ${ }^{1}$ \\ Theory Division, CERN, CH-1211, Geneva 23, Switzerland
}

\begin{abstract}
We study some aspects of dilatonic domain walls in relation to the idea on the noncompact internal space. We find that the warp factor in the spacetime metric increases as one moves away from the domain wall for all the supersymmetric dilatonic domain wall solutions obtained from the (intersecting) BPS branes in string theories through toroidal compactifications, unlike the case of the Randall-Sundrum model. On the other hand, when the dilaton coupling parameter $a$ for the $D$-dimensional extreme dilatonic domain wall takes the values $a^{2}<4 /(D-2)^{2}$, the Kaluza-Klein spectrum of graviton has the same structure as that of the Randall-Sundrum model (and the warp factor decreases in the finite interval around the dilatonic domain wall), thereby implying the possibility of extending the Randall-Sundrum model to the $a^{2}<$ $4 /(D-2)^{2}$ case. We construct fully localized solutions describing extreme dilatonic branes within extreme dilatonic domain walls and the supersymmetric branes within the supersymmetric domain walls of string theories. These solutions are valid in any region of spacetime, not just in the region close to the domain walls.
\end{abstract}

CERN-TH/99-364

November, 1999

\footnotetext{
${ }^{1}$ E-mail: Donam.Youm@cern.ch
} 


\section{Introduction}

It was Kaluza [1] who showed that the Einstein's theory of four-dimensional gravity and the Maxwell's theory of electromagnetism can be unified within the five-dimensional theory of general relativity. In his derivation of his model, Kaluza avoided the question of unobservableness of such extra fifth coordinate simply by assuming that all the fields do not depend on the extra coordinates. Later, Klein [2] explained the unobservableness of and the independence of physical quantities on the extra coordinate by assuming the extra spatial dimension to be too small to be observed. Namely, if one assumes the extra dimension to be a compact manifold, i.e., a circle in the Klein case, then all the fields can be Fourier-expanded and the Fourier coefficients are identified as fields in compactified lower dimensions. Since mass scale of such Fourier modes, called the Kaluza-Klein (KK) modes, is inversely proportional to the size of compactification manifold, if the size of the manifold becomes very small, then the extra massive KK modes become too heavy to be observed. So, in the limit of very small compactification manifold, one can effectively keep only zero modes of the KK spectrum, thereby leading to the original Kaluza's assumption that the physical quantities simply do not depend on the extra coordinate.

This idea of Klein has been dominantly taken within particle physics, including the KK supergravity theories and superstring theories. However, the problem with the Klein's idea on small extra space is that it cannot explain why the extra space has to be markedly different in topology and in size. One of alternative approaches which attempt to address this problem assumes that the extra space is on the same footing as our four-dimensional spacetime, namely the fields depend on the extra space which is now assumed to have infinite extend just like our four-dimensional spacetime or to be not very small. However, the challenge of this approach is to explain why the extra space has not been observed. To avoid this difficulty, it was argued that particles are trapped inside of the four-dimensional hypersurface by a potential well $[3,4,5,6,7,8,9,10,11,12]$ or for topological reason [13]. This idea was also proposed in an attempt to explain smallness of cosmological constant in our four-dimensional universe $[6,7,8]$ and to bring chiral fermions $[14,15,16,17,18,19,20]$ into the theory, which is not achievable from the conventional KK theory with compact internal space.

This old idea on noncompact extra space recently has received revived attention, after it was found out [21, 22, 23] by Randall and Sundrum (RS) that such approach can also be applied to solve the hierarchy problem of particle physics. In the RS model, our four-dimensional world is regarded as a non-dilatonic 3-brane embedded in five-dimensional spacetime. In the RS model, the potential due to gravity is repulsive, unlike the case of the previous works on trapping of matter inside of the four-dimensional hypersurface as discussed in the previous paragraph, because of the 
(exponentially) decreasing (rather than increasing) warp factor in the spacetime metric. This exponentially decreasing warp factor makes the trapping of the gravity inside of the four-dimensional hypersurface possible. In fact, it is also shown that such model with noncompact extra space reproduces [10, 22] Newton's $1 / r^{2}$ law of four-dimensional gravity with experimentally unobservable correction from the (extremely suppressed) contribution of the continuum of massive KK modes [22].

The Ansatz for the five-dimensional spacetime metric used in the RS model is the one with the warp factor, where the four-dimensional part has the conformal factor depending on the extra spatial coordinate. So, such metric can also be regarded as the metric of five-dimensional non-dilatonic domain wall, which is supported by the cosmological constant in five-dimensions. In this paper, we consider the case of dilatonic domain walls, where the cosmological constant term in the action is multiplied by the dilaton factor, with an arbitrary dilaton coupling parameter $a$ in any spacetime dimensions $D$. (Some aspects of dilatonic generalization of the RS model are also studied, for example, in Refs. [24, 25, 26].) We study trapping of matter near the domain wall and the KK spectrum of the graviton in the dilatonic domain wall background. We find that for the supersymmetric dilatonic domain walls that can be obtained by toroidally compactifying the (intersecting) BPS branes in string theories, the warp factor in the metric increases as one moves away from the domain wall. So, the RS-type scenario cannot be realized within such dilatonic domain walls. This is along the same line as the previous works on supergravity embeddings [27, 28, 29, 30] of the RS model. Namely, the BPS domain wall solutions in five-dimensional supergravity theories that have been constructed so far have exponentially increasing warp factor (as oppose to the exponentially decreasing warp factor of the RS model) ${ }^{2}$. This is shown explicitly first time in Ref. [30] within domain wall solutions in five-dimensional gauged $N=2$ supergravity. On the other hand, we find that when the dilaton coupling parameter takes the values $a^{2}<4(D-1) /(D-2)^{2}$ the warp factor decreases in finite interval around the domain wall. Particularly when $a^{2}<4 /(D-2)^{2}$, the potential term in the Schrödinger equation describing the small fluctuation of the spacetime metric around the Minkowski sub-spacetime takes the qualitatively same form as that of the RS model, thereby implying the possibility of the extension of the RS model to the $a^{2}<4 /(D-2)^{2}$ case. Note, this cannot be obtained from the (intersecting) BPS branes in string theories through toroidal compactifications. We also construct fully localized solutions describing various extreme solitons living inside of the dilatonic domain walls, by applying the formalism studied in Ref. [31]. These solutions are valid for any region in spacetime, not just in the region close to the domain walls.

\footnotetext{
${ }^{2}$ I would like to thank Prof. Kallosh for bringing this point to my attention and giving suggestions for better presentation of the results in this paper, after the first version of the paper appeared on the preprint archive.
} 
The paper is organized as follows. In section 2, we discuss aspects of dilatonic domain wall solution, especially in relation to the idea on noncompact internal space. In section 3 , we construct fully localized solutions describing extreme dilatonic branes within the extreme dilatonic domain walls. In section 4, we construct fully localized supergravity solutions describing the BPS branes within the BPS domain walls in string theories.

\section{Dilatonic Domain Walls}

In this section, we discuss solution for a general dilatonic domain wall in $D$-dimensional spacetime and its role as an alternative to the conventional KK theory with noncompact internal space.

We start by summarizing a general dilatonic $p$-brane solution in $D$ spacetime dimensions, since we are interested in obtaining solutions describing branes inside of the worldvolume of domain walls. The Einstein-frame action for the dilatonic $p$-brane with the dilaton coupling parameter $a_{p}$ in $D$ spacetime dimensions is

$$
S_{p}^{E}=\frac{1}{2 \kappa_{D}^{2}} \int d^{D} x \sqrt{-g^{E}}\left[\mathcal{R}_{g^{E}}-\frac{4}{D-2}(\partial \phi)^{2}-\frac{1}{2 \cdot(p+2) !} e^{2 a_{p} \phi} F_{p+2}^{2}\right]
$$

where $\kappa_{D}$ is the $D$-dimensional Einstein gravitational constant, $\phi$ is the dilaton, $F_{p+2}=$ $d A_{p+1}$ is the field strength of the $(p+1)$-form potential $A_{p+1}$. The solution to the field equations of the Einstein-frame action (1) for the extreme dilatonic $p$-brane with the longitudinal coordinates $\mathbf{x}=\left(x_{1}, \ldots, x_{p}\right)$ and the transverse coordinates $\mathbf{y}=$ $\left(y_{1}, \ldots, y_{D-p-1}\right)$ located at $\mathbf{y}=\mathbf{0}$ has the following form:

$$
\begin{aligned}
d s_{E}^{2} & =H_{p}^{-\frac{4(D-p-3)}{(D-2) \Delta_{p}}}\left[-d t^{2}+d x_{1}^{2}+\cdots+d x_{p}^{2}\right]+H_{p}^{\frac{4(p+1)}{(D-2) \Delta_{p}}}\left[d y_{1}^{2}+\cdots+d y_{D-p-1}^{2}\right] \\
e^{2 \phi} & =H_{p}^{\frac{(D-2) a_{p}}{\Delta_{p}}}, \quad A_{t x_{1} \ldots x_{p}}=1-H_{p}^{-1}
\end{aligned}
$$

where

$$
H_{p}=1+\frac{Q_{p}}{|\mathbf{y}|^{D-p-3}}, \quad \Delta_{p}=\frac{(D-2) a_{p}^{2}}{2}+\frac{2(p+1)(D-p-3)}{D-2} .
$$

For general values of the dilaton coupling parameter $a_{p}$, the extreme solution (2) is not supersymmetric. Only the extreme solutions with the specific values of the dilaton coupling parameter $a_{p}$ are supersymmetric. For the consistently truncated supergravity action of the form (1) obtained by compactifying the eleven-dimensional supergravity on $\left(S^{1}\right)^{11-D}$ has the dilaton coupling $a_{p}$ such that $\Delta_{p}=4 / N$ with a positive integer $N[32,33]$. Here, the field strength $F_{p+2}$ is a linear combination of $N$ original $(p+2)$-form field strengths with the same Page charges and the dilaton $\phi$ is a linear-combination of the original (dilatonic) scalars. The extreme dilatonic $p$-brane 
solution (2) with $\Delta_{p}=4 / N$ preserves at least $2^{-N}$ of the maximal symmetry. In other words, all the dilatonic $p$-brane solutions obtained by toroidally compactifying intersecting $N$ numbers of BPS branes in string theories with equal charges have the form (2) with $\Delta_{p}=4 / N$. Note, in this case, the "dilaton" $\phi$ in Eqs. (1) and (2) is rather a linear combination of the dilaton and other scalars in string theory.

The dilatonic domain wall with the dilaton coupling parameter $a$ in $D$ spacetime dimensions corresponds to the $p=D-2$ case of the general dilatonic $p$-brane discussed in the above. Namely, a domain wall in $D$ spacetime dimensions can be regarded as a $(D-2)$-brane, electrically charged under the $(D-1)$-form potential $A_{D-1}$. By applying a Poincaré dualization, one can replace the $D$-form field strength $F_{D}=d A_{D-1}$ by the cosmological constant $\Lambda$. So, the action (1) with $p=D-2$ can be rewritten as

$$
S_{\mathrm{DW}}^{E}=\frac{1}{2 \kappa_{D}^{2}} \int d^{D} x \sqrt{-g^{E}}\left[\mathcal{R}_{g^{E}}-\frac{4}{D-2}(\partial \phi)^{2}+e^{-2 a \phi} \Lambda\right] .
$$

The Einstein-frame solution to the field equations of the action (4) for the extreme dilatonic domain wall located at $y=0$ has the following form:

$$
\begin{aligned}
d s_{E}^{2} & =H^{\frac{4}{(D-2) \Delta}}\left[-d t^{2}+d x_{1}^{2}+\cdots+d x_{D-2}^{2}\right]+H^{\frac{4(D-1)}{(D-2) \Delta}} d y^{2}, \\
e^{2 \phi} & =H^{\frac{(D-2) a}{\Delta}}, \quad A_{t x_{1} \ldots x_{D-2}}=1-H^{-1}
\end{aligned}
$$

where

$$
H=1+Q|y|, \quad \Delta=\frac{(D-2) a^{2}}{2}-\frac{2(D-1)}{D-2} .
$$

Here, the parameter $Q$ in the harmonic function $H$ is related to the cosmological constant $\Lambda$ in the action (4) through

$$
\Lambda=-\frac{2 Q^{2}}{\Delta}
$$

As mentioned in the previous paragraph, the supersymmetric ${ }^{3}$ dilatonic domain wall solution (5) that can be obtained by compactifying (intersecting) BPS branes in string theories on a torus has $\Delta=4 / N$. On the other hand, one can also obtain supersymmetric dilatonic domain wall solutions through different compactification procedure. For example, by compactifying the supersymmetric $D^{\prime}$-dimensional dilatonic $p$-brane action (1) with $\Delta_{p}=4 / N$ on $S^{D^{\prime}-p-2}[34,35]$, one has the action (4) with $a$ and $\Delta$ given by:

$$
|a|=\frac{2}{p} \sqrt{\frac{2\left(D^{\prime}-2\right)-N(p+1)\left(D^{\prime}-p-3\right)}{2\left(D^{\prime}-p-2\right)-N\left(D^{\prime}-p-3\right)}},
$$

\footnotetext{
${ }^{3}$ Strictly speaking, supersymmetry nature of domain wall solutions cannot be fully answered until one considers full supergravity action with non-constant dilaton field (which makes the wall) and the explicit form of superpotential.
} 


$$
\Delta=-\frac{4\left(D^{\prime}-p-3\right)}{2\left(D^{\prime}-p-2\right)-N\left(D^{\prime}-p-3\right)} .
$$

Here, $D$ in Eqs. (4) - (6) is given by $D=p+2$ for this type of compactification.

Note, unlike the case of other type of branes, one can always remove the constant term in the above harmonic function $H$ for the domain wall by applying the coordinate translation along the $y$-direction, and the solution can be written with different powers of harmonic function through the coordinate transformations [36]. In this paper we choose the transverse coordinate such that the solution has the standard form that would be obtained by taking the $p=D-2$ limit of dilatonic $p$-brane solutions.

In general, matter in the $D$-dimensional spacetime with the following form of metric with the warp factor $W(z)$ :

$$
\hat{g}_{\hat{\mu} \hat{\nu}} d x^{\hat{\mu}} d x^{\hat{\nu}}=W(z)\left[-d t^{2}+d x_{1}^{2}+\cdots+d x_{D-2}^{2}\right]+d z^{2}
$$

is trapped inside of the $(D-1)$-dimensional sub-spacetime with the coordinates $\left(x^{\mu}\right)=$ $\left(t, x_{1}, \ldots, x_{D-2}\right)$ for a suitable form of the warp factor $W(z)$. To see [7] this classically, we consider a particle with the $D$-momentum $P=\left(P^{0}, P^{i}, P^{z}\right)$ moving in this spacetime. The Killing vectors for this spacetime are $\partial / \partial t$ and $\partial / \partial x^{i}(i=1, \ldots, D-2)$. So, the constants of motion for the particle are

$$
\begin{aligned}
& E=-\left(P, \frac{\partial}{\partial t}\right)=-P^{\hat{\mu}} \hat{g}_{\hat{\mu} \hat{\nu}}\left(\frac{\partial}{\partial t}\right)^{\hat{\nu}}=P^{0} W(z), \\
& p^{i}=\left(P, \frac{\partial}{\partial x^{i}}\right)=P^{\hat{\mu}} \hat{g}_{\hat{\mu} \hat{\nu}}\left(\frac{\partial}{\partial x^{i}}\right)^{\hat{\nu}}=P^{i} W(z) .
\end{aligned}
$$

The invariant mass $M$ of the particle in this spacetime is defined as

$$
-M^{2}=P^{\hat{\mu}} \hat{g}_{\hat{\mu} \hat{\nu}} P^{\hat{\nu}}=-W(z)\left(P^{0}\right)^{2}+W(z)\left(P^{i}\right)^{2}+\left(P^{z}\right)^{2} .
$$

Therefore, the momentum $P^{z}$ of the particle in the direction $z$ of the extra space is given by

$$
P^{z}=\sqrt{E^{2} W^{-1}-M^{2}-\mathbf{p}^{2} W^{-1}},
$$

where $\mathbf{p}=\left(p^{i}\right)$. The particle is trapped within the $(D-1)$-dimensional spacetime, i.e., the motion of the particle along the $z$-direction is restricted inside of a finite interval around $z=0$, if the energy $E$ of the particle is bounded above by the potential due to gravity:

$$
E<\sqrt{M^{2} W(z)+\mathbf{p}^{2}}
$$

One can bring the spacetime metric in Eq. (5) for the dilatonic domain wall to the form (9) by redefining the transverse coordinate in the following way:

$$
z=\operatorname{sgn}(y) \frac{(D-2) \Delta}{2(D-1)+(D-2) \Delta} Q^{-1}\left[(1+Q|y|)^{\frac{2(D-1)+(D-2) \Delta}{(D-2) \Delta}}-1\right]
$$




$$
=\operatorname{sgn}(y) \frac{a^{2}(D-2)^{2}-4(D-1)}{a^{2}(D-2)^{2}} Q^{-1}\left[(1+Q|y|)^{\frac{a^{2}(D-2)^{2}}{a^{2}(D-2)^{2}-4(D-1)}}-1\right] .
$$

Then, the dilatonic domain wall metric takes the form (9) with the warp factor given by

$$
\begin{aligned}
W(z) & =\left(1+\frac{2(D-1)+(D-2) \Delta}{(D-2) \Delta} Q|z|\right)^{\frac{4}{2(D-1)+(D-2) \Delta}} \\
& =\left(1+\frac{a^{2}(D-2)^{2}}{a^{2}(D-2)^{2}-4(D-1)} Q|z|\right)^{\frac{8}{a^{2}(D-2)^{2}}}
\end{aligned}
$$

This warp factor for the dilatonic domain wall metric monotonically increases as one moves away from the wall and goes to infinity as $|z| \rightarrow \infty$, if $a^{2}>4(D-1) /(D-2)^{2}$, i.e., $\Delta>0$. (Note, this case includes the supersymmetric dilatonic domain walls obtained from the (intersecting) BPS branes in string theories through toroidal compactifications, in which $\Delta=4 / N$.) So, matter inside of $D$-dimensional spacetime of the dilatonic domain wall will always be trapped within the $(D-1)$-dimensional subspacetime by gravity, even if the extra space with the coordinate $z$ can have an infinite extend. This mechanism provides with an alternative to the ordinary KK theories where the spacetime is assumed to be of the form of the direct product $M_{d} \times K$ of the $d$-dimensional spacetime $M_{d}$ and some compact space $K$. On the other hand, when $a^{2}<4(D-1) /(D-2)^{2}$, i.e., $\Delta<0$, the warp factor $W(z)$ decreases at a finite interval $|z|<\left[4(D-1) /\left(a^{2}(D-2)^{2}\right)-1\right] Q^{-1}$ around the domain wall, thereby providing with the repulsive potential to matter. (Note, in this case, the spacetime metric (9) with the warp factor (15) is not well-defined in the region $|z|>\left[4(D-1) /\left(a^{2}(D-2)^{2}\right)-1\right] Q^{-1}$.) However, in this case gravity is trapped within the domain wall for sufficiently small values of $\Delta$, i.e., $\Delta<-2$, as will be discussed in the following.

This trapping of matter due to gravitational potential well can also be seen [11] by considering the Klein-Gordon equation $\nabla^{2} \Psi=M^{2} \Psi$ for a massive scalar $\Psi$ in the background of dilatonic domain wall (9) with the warp factor (15). If we let $\Psi=W^{(3-D) / 2}(z) \phi\left(x^{\mu}\right)$, then the above Klein-Gordon equation takes the following form of the Klein-Gordon equation for a scalar $\phi\left(x^{\mu}\right)$ in $(D-1)$-dimensional flat spacetime with varying mass as one moves along the direction perpendicular to the domain wall:

$$
\eta^{\mu \nu} \partial_{\mu} \partial_{\nu} \phi=\left[M^{2} W(z)+\frac{D-3}{2} W^{\prime \prime}(z)\right] \phi
$$

where the prime denotes derivative with respect to $z$. So, if $a^{2}>4(D-1) /(D-2)^{2}$, i.e., $\Delta>0$, then the mass of the $(D-1)$-dimensional scalar $\phi\left(x^{\mu}\right)$ increases as one moves away from the domain wall, implying that the scalar $\phi$ is in the attractive potential well centered around $z=0$. When $a^{2}<4(D-1) /(D-2)^{2}$, i.e., $\Delta<0$, the minimum of the 
potential well is rather away from the wall, i.e., at $|z|=\left[4(D-1) /\left(a^{2}(D-2)^{2}\right)-1\right] Q^{-1}$, which moves away from the domain wall as the dilaton coupling parameter $a$ approaches zero. This implies the repulsive potential for the $a^{2}<4(D-1) /(D-2)^{2}$ case. As we will discuss in the following paragraph, this critical value $a^{2}=4(D-1) /(D-2)^{2}$ for the dilaton coupling parameter also corresponds to the critical value below [above] which the $\delta$-function potential in the Schrödinger equation satisfied by the metric perturbation becomes attractive [repulsive]. Namely, when $a^{2}>4(D-1) /(D-2)^{2}$, i.e., $\Delta>0$, the minimum of the potential well for a massive particle or massive scalar is located at the domain wall (therefore, matter is trapped due to the attractive potential well) and the $\delta$-function potential in the Schrödinger equation becomes an infinite barrier, but when $a^{2}<4(D-1) /(D-2)^{2}$, i.e., $\Delta<0$, the minima of the potential well is located away from the domain wall (therefore, the potential becomes repulsive) and the $\delta$-function potential in the Schrödinger equation becomes attractive.

In the following, we study the KK spectrum of graviton in the dilatonic domain wall background. We consider the following general metric describing the small fluctuation $h_{\mu \nu}\left(x^{\mu}, u\right)$ of the $(D-1)$-dimensional Minkowski sub-spacetime of the conformally flat $D$-dimensional spacetime of the dilatonic domain wall:

$$
\hat{g}_{\hat{\mu} \hat{\nu}} d x^{\hat{\mu}} d x^{\hat{\nu}}=\lambda(u)\left[\left(\eta_{\mu \nu}+h_{\mu \nu}\right) d x^{\mu} d x^{\nu}+d u^{2}\right],
$$

where $\left|h_{\mu \nu}\right| \ll 1$. The conformally flat form of the spacetime metric (Eq. (17) with $h_{\mu \nu}=0$ ) can be achieved from the dilatonic domain wall metric in Eq. (5) by applying the following coordinate transformation:

$$
u=\operatorname{sgn}(y) \frac{\Delta}{\Delta+2} Q^{-1}\left[(1+Q|y|)^{\frac{\Delta+2}{\Delta}}-1\right],
$$

resulting in the conformally flat metric with the following conformal factor:

$$
\begin{aligned}
\lambda(u) & =\left(1+\frac{\Delta+2}{\Delta} Q|u|\right)^{\frac{4}{(D-2)(\Delta+2)}} \\
& =\left(1+\frac{(D-2)^{2} a^{2}-4}{(D-2)^{2} a^{2}-4(D-1)} Q|u|\right)^{\frac{8}{(D-2)^{2} a^{2}-4}} .
\end{aligned}
$$

The $(\mu, \nu)$-component of the Einstein equations (resulting from the action which also contains the worldvolume action for the domain wall ${ }^{4}$ ) in the transverse traceless Lorentz gauge, i.e., $h^{\mu}{ }_{\mu}=0=\partial^{\mu} h_{\mu \nu}$, is approximated to the first order in the perturbation $h_{\mu \nu}$ to

$$
\left[\square_{x}+\partial_{u}^{2}+\frac{D-2}{2} \frac{\partial_{u} \lambda}{\lambda} \partial_{u}\right] h_{\mu \nu}=0,
$$

\footnotetext{
${ }^{4}$ Note, the scaling symmetry of combined worldvolume and effective bulk actions is broken when $\Delta \neq 4$.
} 
where $\square_{x} \equiv \eta^{\mu \nu} \partial_{\mu} \partial_{\nu}$. We let the metric fluctuation to be of the form $h_{\mu \nu}\left(x^{\mu}, u\right)=$ $e^{i p_{\mu} x^{\mu}} \psi_{\mu \nu}(u)$, where $\eta^{\mu \nu} p_{\mu} p_{\nu}=-m^{2}$. Then, Eq. (20) reduces to

$$
\left[\partial_{u}^{2}+\frac{D-2}{2} \frac{\partial_{u} \lambda}{\lambda} \partial_{u}+m^{2}\right] \psi_{\mu \nu}(u)=0 .
$$

In terms of new field $\tilde{\psi}_{\mu \nu}(u)=\lambda^{(D-2) / 4}(u) \psi_{\mu \nu}(u)$, Eq. (21) takes the following form of the Schrödinger equation

$$
-\frac{d^{2} \tilde{\psi}_{\mu \nu}}{d u^{2}}+V(u) \tilde{\psi}_{\mu \nu}=m^{2} \tilde{\psi}_{\mu \nu}
$$

with potential

$$
V(u)=\frac{D-2}{16}\left[(D-6)\left(\frac{\lambda^{\prime}}{\lambda}\right)^{2}+4 \frac{\lambda^{\prime \prime}}{\lambda}\right],
$$

where the prime denotes differentiation with respect to $u$. By substituting the conformal factor (19) for the dilatonic domain wall metric into this expression, one obtains the following potential

$$
V(u)=-\frac{1+\Delta}{\Delta^{2}} \frac{Q^{2}}{\left(1+\frac{\Delta+2}{\Delta} Q|u|\right)^{2}}+\frac{2 Q}{\Delta} \delta(u) .
$$

This KK potential expression has the similar form as that of the non-dilatonic domain wall of the RS model up to the coefficients. However, there are some qualitative differences in the KK modes depending on the signs of the coefficients in the KK potential (24). In the following, we discuss properties of the KK spectrum for different values of $\Delta$ or $a$.

- $\Delta<-2$ case, i.e., $a^{2}<4 /(D-2)^{2}$ : The coefficient in the first term of the KK potential is positive, the coefficient of the $\delta$-function term is negative and the coefficient in front of $|u|$ in the first term of the KK potential is positive, just as in the case of the RS model ( $a=0$ case). So, the KK spectrum consists of a single normalizable bound state zero mode (identified as the $(D-1)$-dimensional graviton) due to the attractive $\delta$-function potential and the continuum of massive KK modes which asymptote to plane waves as $|u| \rightarrow \infty$ (since $V(u) \rightarrow 0$ as $|u| \rightarrow \infty$ ) and are suppressed as the origin is approached (due to the potential barrier near $u=0)$. This implies that the RS model can be extended to the $a^{2}<$ $4 /(D-2)^{2}$ case. This case cannot be achieved through toroidal compactification of intersecting BPS branes in string theories (since $\Delta=4 / N$ for this case), but can be achieved through the combined toroidal and spherical compactifications (when $N\left(D^{\prime}-p-3\right)>2$ ), as can be seen from $\Delta$ in Eq. (8). However, the issues on supersymmetry of such dilatonic domain walls cannot be fully answered until one considers corresponding full gauged supergravity theory. 
- $-2<\Delta<-1$ case, i.e., $4 /(D-2)^{2}<a^{2}<2 D /(D-2)^{2}$ : The signs of the coefficients are the same as the above case except the coefficient in front of $|u|$, which now becomes negative. So, the first term in the KK potential (24) has the positive minimum at $|z|=0$ and increases monotonically, approaching infinity as $|u|=-Q^{-1} \Delta /(\Delta+2)$ is reached. In this case, one rather has discrete bound states of massive modes with mass gap.

- $-1<\Delta<0$ case, i.e., $2 D /(D-2)^{2}<a^{2}<4(D-1) /(D-2)^{2}$ : The coefficient of the first term in the KK potential becomes negative and the coefficient in front of $|u|$ becomes negative, whereas the coefficient in the $\delta$-function term still remains negative. So, the first term in the KK potential has negative maximum at $|z|=0$ and decreases monotonically, approaching negative infinity as $|u|=$ $-Q^{-1} \Delta /(\Delta+2)$ is reached. In addition to the continuum of massive modes, now there are therefore the continuum of tachyonic modes, implying the instability.

- $\Delta>0$ case, i.e., $a^{2}>4(D-1) /(D-2)^{2}$ : This case includes the case of $\Delta=4 / N$, i.e., dilatonic domain walls obtained from the (intersecting) BPS branes in string theories through toroidal compactifications. The coefficient in the first term is negative, the coefficient in front of $|u|$ is positive and the coefficient in the $\delta$ function term becomes now positive. The first term in the KK potential has the negative minimum at $z=0$ and increases monotonically, approaching zero as $|z| \rightarrow \infty$. So, in this case, now we have discrete bound states of tachyonic modes, in addition to the continuum of massive modes without mass gap. The repulsive $\delta$-function potential at the origin provides with infinite potential barrier at $u=0$. As mentioned previously, this case also corresponds to the case in which matter is trapped within the $(D-1)$-dimensional sub-spacetime by the attractive potential well due to the gravity. So, dilatonic domain walls obtainable from the (intersecting) BPS branes in string theories through toroidal compactification just traps matter inside the domain wall without providing with the RS-type scenario.

\section{Extreme Dilatonic Branes inside of Extreme Dila- tonic Domain Walls}

In this section, we construct fully localized solutions describing extreme dilatonic branes within the worldvolume of extreme dilatonic domain walls. Schematically, the configurations under consideration are given by the following table: 


\begin{tabular}{|l||c|c|c|c|}
\hline & $t$ & $\mathbf{w}$ & $\mathbf{x}$ & $y$ \\
\hline \hline brane & $\bullet$ & $\bullet$ & & \\
\hline domain wall & $\bullet$ & $\bullet$ & $\bullet$ & \\
\hline
\end{tabular}

Here, $t$ is the time coordinate, $\mathbf{w}=\left(w_{1}, \ldots, w_{p}\right)$ is the overall longitudinal coordinates, $\mathbf{x}=\left(x_{1}, \ldots, x_{D-p-2}\right)$ is the relative transverse coordinates for the brane and $y$ is the overall transeverse coordinate. These notations for the coordinates will be followed in this and the next sections.

Such brane configurations are described by the theory which contains the $D$-dimensional graviton $g_{\mu \nu}^{E}(\mu, \nu=0,1, \ldots, D-1)$, the dilaton $\phi,(p+1)$-form potential $A_{p+1}$ with the field strength $F_{p+2}=d A_{p+1}$, which $p$-brane $(p<D-2)$ couples to, and the $(D-1)$ form potential $A_{D-1}$, whose field strength $F_{D}=d A_{D-1}$ dualizes to the cosmological constant term which supports domain wall solution. The Einstein-frame action which describes such theory has the following form:

$$
S_{E}=\frac{1}{2 \kappa_{D}^{2}} \int d^{D} x \sqrt{-g^{E}}\left[\mathcal{R}_{g^{E}}-\frac{4}{D-2}(\partial \phi)^{2}-\frac{1}{2 \cdot(p+2) !} e^{2 a_{p} \phi} F_{p+2}^{2}-\frac{1}{2 \cdot D !} e^{2 a \phi} F_{D}^{2}\right] .
$$

The extreme solution to the field equations of this action describing dilatonic $p$-brane localized inside of the dilatonic domain wall has the following form:

$$
\begin{aligned}
d s_{E}^{2}= & H^{\frac{4}{(D-2) \Delta}}\left[H_{p}^{-\frac{4(D-p-3)}{(D-2) \Delta_{p}}}\left(-d t^{2}+d w_{1}^{2}+\cdots+d w_{p}^{2}\right)\right. \\
& \left.+H_{p}^{\frac{4(p+1)}{(D-2) \Delta_{p}}}\left(d x_{1}^{2}+\cdots+d x_{D-p-2}^{2}\right)\right]+H^{\frac{4(D-1)}{(D-2) \Delta}} H_{p}^{\frac{4(p+1)}{(D-2) \Delta_{p}}} d y^{2}, \\
e^{2 \phi}= & H^{\frac{(D-2) a}{\Delta}} H_{p}^{\frac{(D-2) a_{p}}{\Delta p}}, \\
A_{t w_{1} \ldots w_{p}}= & 1-H_{p}^{-1}, \quad A_{t w_{1} \ldots w_{p} x_{1} \ldots x_{D-p-2}}=1-H^{-1} .
\end{aligned}
$$

Note, the consistency of equations of motion requires [37] that the dilaton coupling parameter $a_{p}$ for the dilatonic $p$-brane is constrained to take the following value:

$$
a_{p}=\frac{p+1}{(D-2)^{2}} \frac{4}{a}
$$

The harmonic functions $H_{p}$ and $H$ for the $p$-brane and the domain wall satisfy the following coupled partial differential equations:

$$
\partial_{y}^{2} H_{p}+H \partial_{\mathbf{x}}^{2} H_{p}=0, \quad \partial_{y}^{2} H=0 .
$$

A general solution for the harmonic function $H$ to the second equation in Eq. (28) has the form $H=1+Q|y|$. However, by applying the coordinate translation $y \rightarrow$ 
$y-\operatorname{sgn}(y) Q^{-1}$ along the $y$-direction, one can remove the constant term in $H$. In this case, the harmonic functions that solve Eq. (28) are given by [31]

$$
H_{p}=1+\frac{Q_{p}}{\left[|\mathbf{x}|^{2}+\frac{4 Q}{9}|y|^{3}\right]^{\frac{3(D-p)-8}{6}}}, \quad H=Q|y| .
$$

Note, these expressions for harmonic functions are valid for any values of $\mathbf{x}$ and $y$. To bring the harmonic function $H$ to the original form, one translates back $y \rightarrow y+$ $\operatorname{sgn}(y) Q^{-1}$ along the $y$-direction, leading to the following expressions for the harmonic functions:

$$
H_{p}=1+\frac{Q_{p}}{\left[|\mathbf{x}|^{2}+\frac{4}{9 Q^{2}}(1+Q|y|)^{3}\right]^{\frac{3(D-p)-8}{6}}}, \quad H=1+Q|y|
$$

Next, the solution to the field equations of the action (4) describing the pp-wave propagating inside of the extreme dilatonic domain wall has the following form:

$$
\begin{aligned}
d s_{E}^{2}= & H^{\frac{4}{(D-2) \Delta}}\left[-d t^{2}+d w^{2}+\left(H_{\mathrm{pp}}-1\right)(d t-d w)^{2}\right. \\
& \left.+d x_{1}^{2}+\cdots+d x_{D-3}^{2}\right]+H^{\frac{4(D-1)}{(D-2) \Delta}} d y^{2} \\
e^{2 \phi}= & H^{\frac{(D-2) a}{\Delta}}
\end{aligned}
$$

where the harmonic functions $H$ and $H_{\mathrm{pp}}$ for the domain wall and the pp-wave satisfy the following coupled partial differential equations:

$$
\partial_{y}^{2} H_{\mathrm{pp}}+H \partial_{\mathbf{x}}^{2} H_{\mathrm{pp}}=0, \quad \partial_{y}^{2} H=0,
$$

where $\mathbf{x}=\left(x_{1}, \ldots, x_{D-3}\right)$, and therefore are given by

$$
H_{\mathrm{pp}}=\frac{Q_{\mathrm{pp}}}{\left[|\mathbf{x}|^{2}+\frac{4}{9 Q^{2}}(1+Q|y|)^{3}\right]^{\frac{3 D-11}{6}}}, \quad H=1+Q|y| .
$$

Note, we have seen in the previous section that matter is trapped within the dilatonic domain wall when $\Delta>0$ and the RS model can be extended to the $\Delta<-2$ case. So, fully localized solutions, presented in this section, describes an extreme brane inside of a dilatonic domain wall which traps matter [which generalizes the RS model] when the dilaton coupling parameter $a$ is such that $\Delta>0[\Delta<-2]$. The former case includes the supersymmetric dilatonic domain walls with $\Delta=4 / N$, which are obtained from the (intersecting) BPS branes in string theories through toroidal compactifications. 


\section{BPS Solitons inside of BPS Domain Walls in String Theories}

In this section, we construct fully localized supergravity solutions describing the BPS solitons inside of the BPS domain walls which are obtained by compactifying intersecting branes in string theories. We shall compactify various partially localized intersecting BPS brane solutions constructed in Ref. [31] to obtain such supergravity solutions. Because the configurations considered in this section (and also those considered in the previous section) describe branes inside of another brane and the dimensionality of the overall transverse space is sufficiently small, the corresponding supergravity solutions are completely localized. Moreover, these solutions describe the field configurations in the region at arbitrary distance from the domain walls.

Generally, the Einstein-frame action for a brane in string theory has the following form:

$$
S_{E}=\frac{1}{2 \kappa_{10}^{2}} \int d^{10} x \sqrt{-G^{E}}\left[\mathcal{R}_{G^{E}}-\frac{1}{2}(\partial \Phi)^{2}-\frac{1}{2 \cdot(p+2) !} e^{2 b \Phi} F_{p+2}^{2}\right],
$$

where $G_{M N}^{E}(M, N=0,1, \ldots, 9)$ is the Einstein-frame spacetime metric, $\Phi$ is the tendimensional dilaton and $F_{p+2}$ is the field strength of the $(p+1)$-form potential $A_{p+1}$. Here, the value of the dilaton coupling parameter $b$ is $-1 / 2$ for the NS-NS 2 -form potential and $-(p-3) / 4$ for the $\mathrm{RR}(p+1)$-form potential. The following string-frame action is obtained through the Weyl rescaling transformation $G_{M N}^{E}=e^{-\Phi / 2} G_{M N}^{s}$ :

$$
S_{s}=\frac{1}{2 \kappa_{10}^{2}} \int d^{10} x \sqrt{-G^{s}}\left[e^{-2 \Phi}\left\{\mathcal{R}_{G^{s}}+4(\partial \Phi)^{2}\right\}-\frac{1}{2 \cdot(p+2) !} F_{p+2}^{2}\right],
$$

for the $\mathrm{RR}(p+1)$-form potential case, and

$$
S_{s}=\frac{1}{2 \kappa_{10}^{2}} \int d^{10} x \sqrt{-G^{s}} e^{-2 \Phi}\left[\mathcal{R}_{G^{s}}+4(\partial \Phi)^{2}-\frac{1}{2 \cdot 3 !} F_{3}^{2}\right],
$$

for the NS-NS 2-form potential case.

We shall compactify some of the longitudinal and the transverse directions of the intersecting brane configurations to obtain the configurations describing the BPS solitons inside of domain walls. Since, generally, the spacetime metric of intersecting brane solutions are diagonal, the KK Ansatz for the string-frame metric is

$$
\left(G_{M N}^{s}\right)=\left(\begin{array}{cc}
g_{\mu \nu}^{s} & 0 \\
0 & g_{m n}
\end{array}\right)
$$

where $g_{\mu \nu}^{s}(\mu, \nu=0,1, \ldots, D-1)$ is the string-frame metric in $D$ spacetime dimensions and $g_{m n}(m, n=1, \ldots, 10-D)$ is the internal metric. The $D$-dimensional dilaton $\phi$ 
is expressed in terms of the ten-dimensional dilaton $\Phi$ and the internal metric $g_{m n}$ as follows:

$$
\phi=\Phi-\frac{1}{4} \ln \operatorname{det} g_{m n} .
$$

After obtaining the string-frame metric $g_{\mu \nu}^{s}$ for a $D$-dimensional solution by using the simple compactification Ansatz (37), one can apply the Weyl-scaling transformation $g_{\mu \nu}^{E}=e^{-\frac{4}{D-2} \phi} g_{\mu \nu}^{s}$ to obtain the Einstein-frame metric $g_{\mu \nu}^{E}$ for the solution. In addition to the metric, the dilaton and the form-potential, the compactified $D$-dimensional action has additional scalars associated with the internal metric $g_{m n}$, unlike the case of dilatonic $p$-branes inside of dilatonic domain walls considered in the previous section. As a consequence, the dilaton coupling parameter for $p$-branes in string theories are not restricted by the constraint in Eq. (27)

Supergravity solutions for branes in string theories have the following convenient properties, which simplify the study of brane solutions in $D<10$ significantly. First, the Einstein-frame solution for the spacetime metric depends only on the spacetime dimensions $D$ and the dimensionality $p$ of the brane, independently of the type of form potential under which brane is charged. Second, in the case of the RR-charged $p$ branes in $D<10$, expressions for the spacetime metric and the $D$-dimensional dilaton are insensitive to their origin in $D=10$. Namely, for example, the spacetime metric and the $D$-dimensional dilaton for RR 2-brane in $D=6$ have the same form whether they are obtained by compactifying one longitudinal direction and three transverse directions of D3-brane or they are obtained by compactifying four transverse directions of D2-brane.

The universal forms of the Einstein-frame metrics for a $p$-brane and a domain wall in string theories in $D<10$ are as follows:

- $p$-brane:

$$
d s_{E}^{2}=H_{p}^{-\frac{D-p-3}{D-2}}\left[-d t^{2}+d x_{1}^{2}+\cdots+d x_{p}^{2}\right]+H_{p}^{\frac{p+1}{D-2}}\left[d y_{1}^{2}+\cdots+d y_{D-p-1}^{2}\right],
$$

where $H_{p}=1+\frac{Q_{p}}{|\mathbf{y}|^{D-p-3}}$. This is the $\Delta_{p}=4$ case of the general dilatonic $p$-brane metric in Eq. (2).

- domain wall ( $p=D-2$ case of the above):

$$
d s_{E}^{2}=H^{\frac{1}{D-2}}\left[-d t^{2}+d x_{1}^{2}+\cdots+d x_{D-2}^{2}\right]+H^{\frac{D-1}{D-2}} d y^{2} .
$$

This is the $\Delta=4$ case of the spacetime metric for a general dilatonic domain wall solution in Eq. (5).

Therefore, the Einstein-frame spacetime metrics for the $D$-dimensional configurations describing the BPS branes inside of the worldvolume of the BPS domain walls in string theories have the following universal forms: 
- The BPS p-brane inside of the BPS domain wall worldvolume:

$$
\begin{aligned}
d s_{E}^{2}= & H^{\frac{1}{D-2}}\left[H_{p}^{-\frac{D-p-3}{D-2}}\left(-d t^{2}+d w_{1}^{2}+\cdots+d w_{p}^{2}\right)\right. \\
& \left.+H_{p}^{\frac{p+1}{D-2}}\left(d x_{1}^{2}+\cdots+d x_{D-p-2}^{2}\right)\right]+H^{\frac{D-1}{D-2}} H_{p}^{\frac{p+1}{D-2}} d y^{2} .
\end{aligned}
$$

The harmonic functions $H$ and $H_{p}$ for the domain wall and the $p$-brane satisfy the coupled partial differential equations (28) and therefore are given by Eq. (29) or Eq. (30).

- The pp-wave propagating inside of the BPS domain wall:

$$
\begin{gathered}
d s_{E}^{2}=H^{\frac{1}{D-2}}\left[-d t^{2}+d w^{2}+\left(H_{\mathrm{pp}}-1\right)(d t-d w)^{2}\right. \\
\left.+d x_{1}^{2}+\cdots+d x_{D-3}^{2}\right]+H^{\frac{D-1}{D-2}} d y^{2} .
\end{gathered}
$$

The harmonic functions $H$ and $H_{\mathrm{pp}}$ for the domain wall and the pp-wave satisfy the coupled partial differential equations (32) and therefore are given by Eq. (33).

The expressions for $D$-dimensional dilaton $\phi$ for various cases are as follows:

- $\mathrm{RR} p$-branes (dimensionally reduced from D-branes): $e^{2 \phi}=H_{p}^{(D-2 p-4) / 4}$.

- magnetic NS $p$-branes with $p=0,1, \ldots, 5$ (dimensionally reduced from NS5-brane in $D=10): e^{2 \phi}=H_{p}^{(D-p-3) / 2}$.

- electric NS $p$-branes with $p=0,1$ (dimensionally reduced from the fundamental string in $D=10): e^{2 \phi}=H_{p}^{-(p+1) / 2}$.

- domain walls compactified from D-branes: $e^{2 \phi}=H^{-D / 4}$.

- domain walls compactified from NS5-brane: $e^{2 \phi}=H^{-1 / 2}$.

The form potential for a $p$-brane has the standard form $A_{t x_{1} \ldots x_{p}}=1-H_{p}^{-1}$. The scalars originated from the internal components of the spacetime metric are different for different types of branes and compactifications.

Whereas the Einstein-frame spacetime metrics have the universal forms (as discussed in the above), the expressions for string-frame spacetime metrics are different for different types of form-fields under which the branes are charged. So, in the following subsections, we present the expressions for the string-frame spacetime metric, as well as the dilaton, for various cases. 


\subsection{BPS solitons within the RR domain walls}

In this subsection, we write down the expressions for the string-frame metrics and the dilatons of supergravity solutions describing various BPS solitons in the worldvolume of the domain wall charged under the RR form-potential.

First, we consider the configurations where the $\mathrm{RR}$ charged $p$-brane is inside of the worldvolume of the RR domain walls. Supergravity solutions for such configurations are obtained by compactifying the partially localized supergravity solutions describing two $\mathrm{D} p$-branes self-intersecting over $(p-2)$-dimensions, $\mathrm{D} p$-brane ending on $\mathrm{D}(p+2)$ brane and $\mathrm{D} p$-brane inside of $\mathrm{D}(p+4)$-brane. The string-frame metric and the dilaton field have the following forms:

$$
\begin{aligned}
d s_{s}^{2}= & H^{-\frac{1}{2}}\left[H_{p}^{-\frac{1}{2}}\left(-d t^{2}+d w_{1}^{2}+\cdots+d w_{p}^{2}\right)+H_{p}^{\frac{1}{2}}\left(d x_{1}^{2}+\cdots+d x_{D-p-2}^{2}\right)\right] \\
& \quad+H^{\frac{1}{2}} H_{p}^{\frac{1}{2}} d y^{2} \\
e^{2 \phi}= & H^{-\frac{D}{4}} H_{p}^{\frac{D-2 p-4}{4}}
\end{aligned}
$$

where here and in the following the harmonic functions $H$ and $H_{p}$ for the domain wall and the $p$-brane are given by Eq. (29) or Eq. (30).

Second, the supergravity solutions describing the electric NS-NS charged $p$-brane $(p=0,1)$ inside of the worldvolume of the RR domain wall are obtained by compactifying the partially localized supergravity solution describing the fundamental string ending on D-branes. The string-frame metric and the dilaton have the following forms:

$$
\begin{aligned}
d s_{s}^{2} & =H^{-\frac{1}{2}}\left[H_{p}^{-1}\left(-d t^{2}+d w_{1}^{2}+\cdots+d w_{p}^{2}\right)+d x_{1}^{2}+\cdots+d x_{D-p-2}^{2}\right]+H^{\frac{1}{2}} d y^{2}, \\
e^{2 \phi} & =H^{-\frac{D}{4}} H_{p}^{-\frac{p+1}{2}} .
\end{aligned}
$$

Third, the supergravity solutions describing the magnetic NS-NS charged $p$-brane $(p=0,1, \ldots, 5)$ inside of the worldvolume of the $\mathrm{RR}$ domain wall are obtained by compactifying the partially localized supergravity solution describing D-branes ending on NS5-brane. The string-frame metric and the dilaton have the following forms:

$$
\begin{aligned}
d s_{s}^{2} & =H^{-\frac{1}{2}}\left[-d t^{2}+d w_{1}^{2}+\cdots+d w_{p}^{2}+H_{p} d x^{2}\right]+H^{\frac{1}{2}} H_{p} d y^{2} \\
e^{2 \phi} & =H^{-\frac{D}{4}}
\end{aligned}
$$

where $D=p+3$.

Fourth, the supergravity solution describing the pp-wave propagating in the worldvolume of the RR domain wall is obtained by compactifying the partially localized supergravity solutions describing the pp-wave propagating in the worldvolume of the D-branes. The string-frame metric and the dilaton have the following forms:

$$
d s_{s}^{2}=H^{-\frac{1}{2}}\left[-d t^{2}+d w^{2}+\left(H_{\mathrm{pp}}-1\right)(d t-d w)^{2}+d x_{1}^{2}+\cdots+d x_{D-3}^{2}\right]+H^{\frac{1}{2}} d y^{2},
$$




$$
e^{2 \phi}=H^{-\frac{D}{4}}
$$

where here and in the following the harmonic functions $H$ and $H_{\mathrm{pp}}$ for the domain wall and the pp-wave are given by Eq. (33).

\subsection{BPS solitons within the NS-NS domain walls}

In this subsection, we write down the expressions for the string-frame metrics and the dilatons of supergravity solutions describing various BPS solitons living in the worldvolume of the domain wall magnetically charged under the NS-NS form-potential.

First, the following string-frame metric and the dilaton of the supergravity solution describing the RR charged BPS $p$-brane inside of the worldvolume of the NS-NS BPS domain wall are obtained by compactifying the partially localized supergravity solution describing the Dp-brane ending on NS5-brane:

$$
\begin{aligned}
d s_{s}^{2} & =H_{p}^{-\frac{1}{2}}\left(-d t^{2}+d w_{1}^{2}+\ldots+d w_{p}^{2}\right)+H_{p}^{\frac{1}{2}}\left(d x_{1}^{2}+\ldots+d x_{D-p-2}^{2}\right)+H H_{p}^{\frac{1}{2}} d y^{2}, \\
e^{2 \phi} & =H^{-\frac{1}{2}} H_{p}^{\frac{D-2 p-4}{4}} .
\end{aligned}
$$

where $p=0,1, \ldots, 5$.

Second, the following string-frame metric and the dilaton of the supergravity solution describing the electric NS-NS charged $p$-brane $(p=0,1)$ in the worldvolume of the NS-NS domain wall are obtained by compactifying the partially localized supergravity solution describing the fundamental string inside of the worldvolume of the NS5-brane:

$$
\begin{aligned}
d s_{s}^{2} & =H_{p}^{-1}\left(-d t^{2}+d w_{1}^{2}+\cdots+d w_{p}^{2}\right)+d x_{1}^{2}+\cdots+d x_{D-p-2}^{2}+H d y^{2}, \\
e^{2 \phi} & =H^{-\frac{1}{2}} H_{p}^{-\frac{p+1}{2}} .
\end{aligned}
$$

Third, the following string-frame metric and the dilaton of the supergravity solution describing the magnetic NS-NS charged $p$-brane $(p=0,1, \ldots, 3)$ in the worldvolume of the NS-NS domain wall are obtained by compactifying the partially localized supergravity solution describing two NS5-branes intersecting over 3 dimensions:

$$
\begin{aligned}
d s_{s}^{2} & =-d t^{2}+d w_{1}^{2}+\cdots+d w_{p}^{2}+H_{p}\left(d x_{1}^{2}+\cdots+d x_{D-p-2}^{2}\right)+H H_{p} d y^{2}, \\
e^{2 \phi} & =H^{-\frac{1}{2}} H_{p}^{\frac{D-p-3}{2}} .
\end{aligned}
$$

Fourth, the following string-frame metric and the dilaton for the supergravity solution describing the pp-wave propagating inside of the NS-NS domain wall are obtained by compactifying the partially localized supergravity solution describing the pp-wave propagating inside of the NS5-brane:

$$
\begin{aligned}
d s_{s}^{2} & =-d t^{2}+d w^{2}+\left(H_{\mathrm{pp}}-1\right)(d t-d w)^{2}+d x_{1}^{2}+\cdots+d x_{D-3}^{2}+H d y^{2} \\
e^{2 \phi} & =H^{-\frac{1}{2}}
\end{aligned}
$$




\section{References}

[1] T. Kaluza, "Zum Unitätsproblem der Physik," Akad. Wiss. Phys. Math. K1 (1921) 966.

[2] O. Klein, "Quantentheorie und fünfdimensionale Relativitätstheorie," Zeits. Phys. 37 (1926) 895.

[3] D.W. Joseph, "Coordinate covariance and the particle spectrum," Phys. Rev. 126 (1962) 319.

[4] K. Akama, "Pregeometry. (Talk)," In *Tokyo 1981, Proceedings, Quark and Lepton Physics*, 342-346, hep-th/0001113.

[5] V.A. Rubakov and M.E. Shaposhnikov, "Do we live inside a domain wall?," Phys. Lett. B125 (1983) 136.

[6] V.A. Rubakov and M.E. Shaposhnikov, "Extra space-time dimensions: towards a solution to the cosmological constant problem," Phys. Lett. B125 (1983) 139.

[7] M. Visser, "An exotic class of Kaluza-Klein models," Phys. Lett. B159 (1985) 22, hepth/9910093.

[8] E.J. Squires, "Dimensional reduction caused by a cosmological constant," Phys. Lett. B167 (1986) 286.

[9] P. Laguna-Castillo and R.A. Matzner, "Surfaces of discontinuity in five-dimensional Kaluza-Klein cosmologies," Nucl. Phys. B282 (1987) 542.

[10] M. Gogberashvili, "Hierarchy problem in the shell-universe model," hep-ph/9812296.

[11] M. Gogberashvili, "Four dimensionality in non-compact Kaluza-Klein model," hep$\mathrm{ph} / 9904383$.

[12] M. Gogberashvili, "Gravitational trapping for extended extra dimension," hep$\mathrm{ph} / 9908347$.

[13] G.W. Gibbons and D.L. Wiltshire, "Space-Time as a membrane in higher dimensions," Nucl. Phys. B287 (1987) 717.

[14] C. Wetterich, "Dimensional reduction of fermions in generalized gravity," Nucl. Phys. B242 (1984) 473.

[15] M. Gell-Mann and B. Zwiebach, "Curling up two spatial dimensions With $S U(1,1) / U(1), "$ Phys. Lett. B147 (1984) 111.

[16] C. Wetterich, "The cosmological constant and noncompact internal spaces in KaluzaKlein theories," Nucl. Phys. B255 (1985) 480.

[17] M. Gell-Mann and B. Zwiebach, "Dimensional reduction of space-time induced by nonlinear scalar dynamics and noncompact extra dimensions," Nucl. Phys. B260 (1985) 569.

[18] B. Mcinnes, "Global Aspects Of Spontaneous Compactification," Class. Quant. Grav. 2 (1985) 661.

[19] S. Randjbar-Daemi and C. Wetterich, "Kaluza-Klein solutions with noncompact internal spaces," Phys. Lett. B166 (1986) 65. 
[20] C. Wetterich, "Fermion chirality from higher dimensions and Kaluza-Klein theories with noncompact internal space," Lectures given at 2nd Jerusalem Winter School on Theoretical Physics, Jerusalem, Israel, Dec 28, 1984 - Jan 4, 1985.

[21] L. Randall and R. Sundrum, "A large mass hierarchy from a small extra dimension," Phys. Rev. Lett. 83 (1999) 3370, hep-ph/9905221.

[22] L. Randall and R. Sundrum, "An alternative to compactification," hep-th/9906064.

[23] J. Lykken and L. Randall, "The shape of gravity," hep-th/9908076.

[24] A. Kehagias, "Exponential and power-law hierarchies from supergravity," hepth/9906204.

[25] E. Halyo, "Localized gravity on branes in anti-de Sitter spaces," hep-th/9909127.

[26] C. Grojean, J. Cline and G. Servant, "Supergravity inspired warped compactifications and effective cosmological constants," hep-th/9910081.

[27] K. Behrndt and M. Cvetič, "Supersymmetric domain wall world from $D=5$ simple gauged supergravity," hep-th/9909058.

[28] K. Skenderis and P.K. Townsend, "Gravitational stability and renormalization-group flow," hep-th/9909070.

[29] A. Chamblin and G.W. Gibbons, "Supergravity on the brane," hep-th/9909130.

[30] R. Kallosh, A. Linde and M. Shmakova, "Supersymmetric multiple basin attractors," JHEP 11 (1999) 010, hep-th/9910021.

[31] D. Youm, "Partially localized intersecting BPS branes," hep-th/9902208.

[32] H. Lu, C.N. Pope, E. Sezgin and K.S. Stelle, "Stainless super p-branes," Nucl. Phys. B456 (1995) 669, hep-th/9508042.

[33] H. Lu and C.N. Pope, "p-brane solitons in maximal supergravities," Nucl. Phys. B465 (1996) 127, hep-th/9512012.

[34] H.J. Boonstra, K. Skenderis and P.K. Townsend, "The domain wall/QFT correspondence," JHEP 01 (1999) 003, hep-th/9807137.

[35] K. Behrndt, E. Bergshoeff, R. Halbersma and J.P. van der Schaar, "On domainwall/QFT dualities in various dimensions," Class. Quant. Grav. 16 (1999) 3517, hepth/9907006.

[36] E. Bergshoeff and J.P. van der Schaar, "On M-9-branes," Class. Quant. Grav. 16 (1999) 23, hep-th/9806069.

[37] R. Argurio, F. Englert and L. Houart, "Intersection rules for p-branes," Phys. Lett. B398 (1997) 61, hep-th/9701042. 